\title{
GATA4 negatively regulates bone sialoprotein expression in osteoblasts
}

\author{
Insun Song ${ }^{1, *}$, Byung-chul Jeong ${ }^{2,3}$, Yong Jun Choi ${ }^{4}$, Yoon-Sok Chung ${ }^{4}$ E Nacksung Kim ${ }^{2,3, *}$ \\ ${ }^{1}$ Shool of Biological Sciences, Seoul National University, Seoul 08826, Departments of ${ }^{2}$ Pharmacology and ${ }^{3}$ Biomedical Sciences, Chonnam \\ National University Medical School, Gwangju 61469, ${ }^{4}$ Department of Endocrinology, Ajou University, Suwon 16499, Korea
}

GATA4 has been reported to act as a negative regulator in osteoblast differentiation by inhibiting the Dlx5 transactivation of Runx2 via the attenuation of the binding ability of Dlx5 to the Runx2 promoter region. Here, we determine the role of GATA4 in the regulation of bone sialoprotein (Bsp) in osteoblasts. We observed that the overexpression of Runx2 or Sox9 induced the Bsp expression in osteoblastic cells. Silencing GATA4 further enhanced the Runx2- and Sox9mediated Bsp promoter activity, whereas GATA4 overexpression down-regulated Bsp promoter activity mediated by Runx2 and Sox9. GATA4 also interacted with Runx2 and Sox9, by attenuating the binding ability of Runx2 and Sox9 to the Bsp promoter region. Our data suggest that GATA4 acts as a negative regulator of Bsp expression in osteoblasts. [BMB Reports 2016; 49(6): 343-348]

\section{INTRODUCTION}

Bone remodeling is controlled by osteoblasts and osteoclasts, which regulate the bone formation and bone resorption, respectively $(1,2)$. The balance between osteoblastic and osteoclastic activities is important for bone homeostasis, while an imbalance leads to bone diseases such as osteoporosis.

Runx2 is involved in mesenchymal condensation, osteoblast differentiation, chondrocyte hypertrophy, and vascular invasion of the developing skeleton from mesenchymal stem cells $(3,4)$. Cleidocranial dysplasia, the defective bone formation disease in humans, results from Runx2 haploinsufficiency $(4,5)$. Runx2 is controlled by various transcription factors such as

*Corresponding authors. Nacksung Kim, Tel: +82-62-220-4418; Fax: +82-62-223-4018; E-mail: nacksung@jnu.ac.kr, Insun Song, Tel: +82-62-220-4418; Fax: +82-62-223-4018; E-mail: song1009@ gmail.com

http://dx.doi.org/10.5483/BMBRep.2016.49.6.032

Received 15 February 2016, Revised 11 March 2016, Accepted 11 March 2016

Keywords: Bone sialoprotein, GATA4, Gene regulation, Runx2, Sox9
Dlx5 and TAZ (6-8). During osteoblast differentiation, it induces the expression of osteoblast marker genes, including alkaline phosphatase $(A L P)$, collagen type I, osteopontin, bone sialoprotein (Bsp), and osteocalcin (OCN) (9).

Sox9 is a member of the Sox family of transcription factors containing a highly conserved high mobility group (HMG) domain (10). To date, about 20 Sox proteins have been identified in mice and humans which are grouped into A through $\mathrm{H}$, based on their structural homology outside of their HMG boxes (11). Sox9 presents a consensus DNA-binding sequence (AACAAT) and regulates various biological processes (10). Sox9 is involved in chondrogenic differentiation and extracellular matrix (ECM) deposition (12).

GATA4 is a member of the GATA family of zinc finger transcription factors and plays a role in cardiac development and adult cardiac hypertrophy. GATAs present a consensus DNA-binding sequence (A/T)GATA(A/G) and regulate various biological processes. GATA1, 2, and 3 are expressed in hematopoietic stem cells, whereas GATA4, 5, and 6 are expressed in mesoderm- and endoderm-derived tissues $(13,14)$. Among them, GATA4 plays various roles through interactions with regulatory proteins such as $\mathrm{p} 300, \mathrm{RXR} \alpha$, and SRF (15).

Bsp, an extracellular matrix protein, plays a critical role in osteoblast differentiation involved in cementum integrity, and is essential for periodontal function (16). Bsp belongs to the small Integrin Binding Ligand N-linked Glycoprotein (SIBLING) family, involved in mineralized tissues of the skeleton and dentition (16-18). Like other SIBLING proteins, Bsp is multifunctional, with roles in cell attachment and migration, cell signaling, collagen binding, and hydroxyapatite nucleation (16). Hunter and Goldberg demonstrated that in vitro, Bsp is a positive regulator of hydroxyapatite precipitation (19). Mice that were null for the $B s p$ gene $(B s p-/-)$ presented delays in bone growth, mineralization, and deficiency in acellular cementum and periodontal attachment $(16,20)$.

Although Bsp is one of the important genes for bone homeostasis, the regulatory mechanism of Bsp expression remains unclear. When we analyzed the $B s p$ promoter region sequence, we identified putative binding sites for Runx2, Sox9, and GATA4. Therefore, in the present study, the role of Runx2, Sox9, and GATA4 in the regulation of Bsp gene expression has been investigated.

ISSN: 1976-670X (electronic edition)

Copyright (c) 2016 by the The Korean Society for Biochemistry and Molecular Biology

(c) This is an open-access article distributed under the terms of the Creative Commons Attribution Non-Commercial License (http://creativecommons.org/licenses/by-nc/4.0) which permits unrestricted non-commercial use, distribution, and reproduction in any medium, provided the original work is properly cited. 


\section{RESULTS}

\section{GATA4 regulates Bsp promoter activities mediated by Runx2 and Dlx5}

Runx2 is important for the regulation of osteoblastic genes such as Bsp. Therefore, we investigated the role of Runx2 in $B s p$ expression by transient transfection assays using a Bsp luciferase reporter vector containing the $1.5 \mathrm{~Kb}$ promoter region of Bsp. This $1.5 \mathrm{~Kb}$ promoter region contains three putative Runx2-binding sites and one putative GATA-binding site (Fig. 1A) (7). Consistent with previous reports (21), Runx2 strongly induced the activity of the Bsp promoter (Fig. 1B). Runx2-mediated Bsp promoter activity was further enhanced by GATA4 knockdown using a shGATA4 construct, while GATA4 overexpression significantly attenuated the Runx2mediated Bsp promoter activity.

Since the $B s p 1.5 \mathrm{~Kb}$ promoter region also contains a putative Dlx5-binding site, we examined the effect of GATA4 on Dlx5-mediated activation of the $B s p$ promoter. Dlx5 induced the activity of the $B s p$ promoter, although activation of the Bsp promoter by Dlx5 was weaker than that mediated by Runx2 (Fig. 1B, C). Similar to Runx2 results, GATA4 overexpression reduced Dlx5-mediated activation of the Bsp promoter, whereas GATA4 knockdown significantly increased the Dlx5-mediated Bsp promoter activity (Fig. 1C). Together,

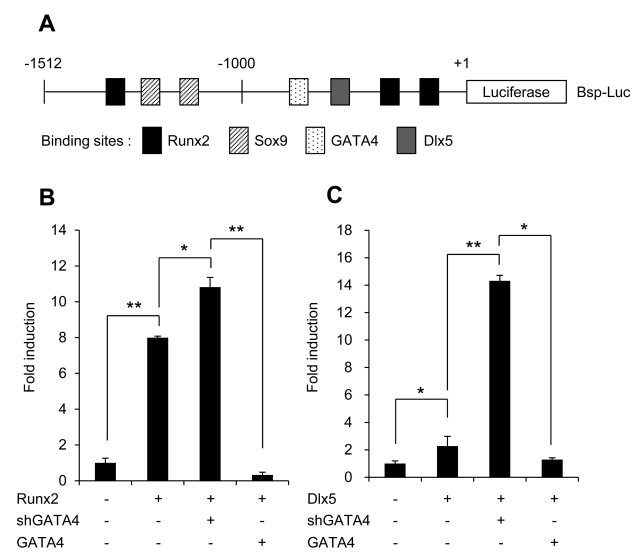

Fig. 1. GATA4 attenuates the Runx2- and Dlx5-mediated activation of the Bsp promoter. (A) Bsp-1.5 kb promoter luciferase reporter (Bsp-1512-Luc) contains putative binding sites for Runx2, Dlx5, Sox9, and GATA4. (B) C2C12 cells were co-transfected with Bsp-1512-Luc luciferase reporter, together with Runx2 and shRNA-GATA4 or GATA4, as indicated. (C) C2C12 cells were co-transfected with the Bsp-1512-Luc luciferase reporter together with DIx5 and shRNA-GATA4 or GATA4, as indicated. Each well was also co-transfected with 20 ng Renilla expression vector to control for transfection efficiency. Luciferase activity was normalized to Renilla activity as expressed by the co-transfected plasmid. Data are presented as mean \pm SD of triplicate samples. Results are representative of at least 3 independent sets of similar experiments. ${ }^{*} \mathrm{P}<0.05,{ }^{*} \mathrm{P}<0.01$ versus control. these results suggest that GATA4 attenuates Runx2- and Dlx5-mediated activation of the Bsp promoter.

\section{Sox9 and Runx2 regulate Bsp expression}

To explore the role of Sox9 in osteoblasts, we examined the expression pattern of Sox9 during osteoblast differentiation. Osteoblast-like MC3T3-E1 cells were cultured in osteogenic medium containing ascorbic acid, $\beta$-glycerophosphate, and bone morphogenetic protein 2 (BMP-2). In reverse transcription polymerase chain reaction (RT-PCR) analysis, the expression of well-known osteogenic marker genes, including Runx2, Bsp, and OCN, was strongly induced during osteoblast differentiation. Sox9 was also steadily expressed in MC3T3-E1 cells during osteoblast differentiation (Fig. 2A), suggesting that Sox9 might play a role in osteoblast differentiation.

Next, we examined the effect of Sox9 on Bsp expression in osteoblasts. Sox9- or Runx2-expressing plasmid was transiently transfected in MC3T3-E1, and Bsp expression was examined by RT-PCR. Bsp expression was increased by overexpression of Sox9 and Runx2 (Fig. 2B). Interestingly, Sox9 and Runx2 mutually increased the $B s p$ gene expression. Similar results were observed when we investigated the effect of Sox9 on Bsp expression at the protein level. Expression levels of Bsp protein were strongly increased by Sox9 or Runx2 in a dosedependent manner in MC3T3-E1 cells (Fig. 2C, D) and $\mathrm{C} \mathrm{H}_{10 \mathrm{~T}} \mathrm{~T}_{1 / 2}$ (Fig. 2E). Collectively, these findings indicate that Sox9 might play a role in the regulation of Bsp expression during osteoblast differentiation.
A

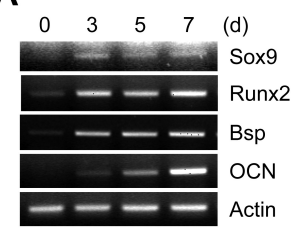

C

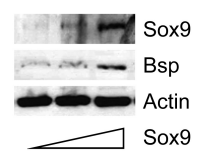

B

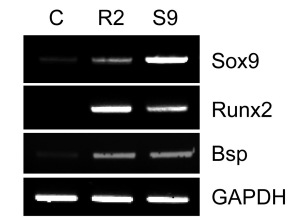

E

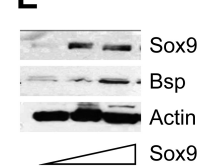

Fig. 2. Sox9 and Runx2 increase Bsp expression. (A) MC3T3-E1 cells were cultured in osteogenic medium containing ascorbic acid and $\beta$-glycerophosphate. Total RNA was collected at each time point. RT-PCR was performed for Sox9 and osteogenic marker genes, including Runx2, Bsp, OCN, and $\beta$-actin for control. (B) MC3T3-E1 cells were transduced with control (pcDNA3.1; C), Runx2 (R2), or Sox9 (S9). After 48 hours, total RNA was collected from each sample. RT-PCR was performed to assess the expression of Sox9, Runx2, Bsp, and GAPDH for control. (C, D) MC3T3-E1 cells were transduced with various concentrations (200, 500, and 1,000 ng) of Sox9 (C) and Runx2 (D). (E) $\mathrm{C}_{3} \mathrm{H}_{10 \mathrm{~T}} \mathrm{~T}_{1 / 2}$ cells were transduced with various concentrations $(200,500$, and 1,000 ng) of Sox9. 
Sox9 and Runx2 synergistically activate the Bsp promoter Since Sox9 overexpression induced Bsp expression in osteoblastic cells, we investigated whether Sox9 directly activates the $B s p$ promoter by transient transfection assay using the Bsp promoter plasmid. Sequence analysis indicated the presence of two putative Sox9-binding sites in the $1.5 \mathrm{~Kb}$ promoter region of $B s p$ (Fig. 1A). Sox9 overexpression significantly enhanced the activity of the $B s p$ promoter in a dose-dependent manner (Fig. 3A). In addition, Sox9 and Runx2 synergistically activated the Bsp promoter (Fig. 3B). Collectively, our data imply that Sox9 directly activates the Bsp promoter, and that Sox9 cooperates with Runx2 to induce Bsp expression during osteoblast differentiation.

GATA4 regulates the $B s p$ promoter activities mediated by Runx2 and Sox 9

Since we observed that GATA4 attenuated the Bsp promoter

A

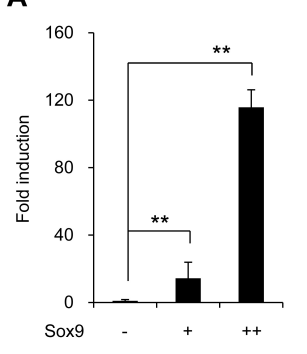

B

C
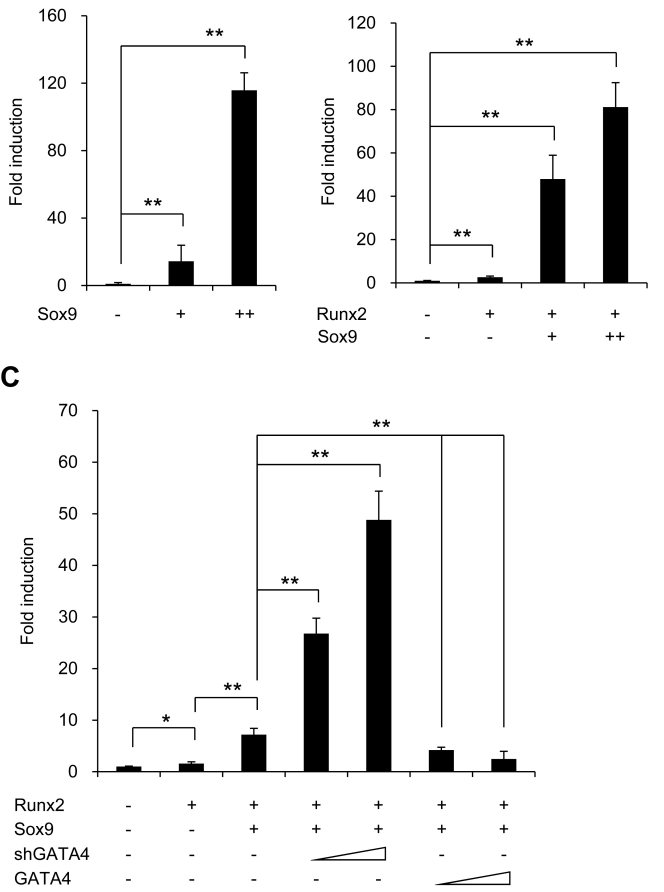

Fig. 3. GATA4 attenuates the Runx2- and Sox9-mediated Bsp activation. (A) $\mathrm{C} 2 \mathrm{C} 12$ cells were co-transfected with the Bsp-1.5 $\mathrm{kb}$ promoter luciferase reporter and Sox9. (B) C2C12 cells were co-transfected with the Bsp-1512-Luc luciferase reporter and Runx2 and Sox9, as indicated. (C) C2C12 cells were co-transfected with the Bsp-1512-Luc luciferase reporter and Runx2, Sox9, and shRNA-GATA4 (25 and $50 \mathrm{ng}$ ) or GATA4 (50 and $100 \mathrm{ng}$ ) as indicated. Luciferase activity was normalized to Renilla activity as expressed by the co-transfected plasmid. Results are representative of at least 3 independent sets of similar experiments. Data are presented as mean \pm SD of triplicate samples. ${ }^{* P}<0.05,{ }^{* * P}<$ 0.01 versus control. activities mediated by Runx2 and Dlx5 (Fig. 1), we next tested whether GATA4 regulates the Bsp promoter activities mediated by Runx2 and Sox9. Consistent with the above mentioned data, combination of Runx2 and Sox9 enhanced the Bsp promoter activity. However, GATA4 overexpression downregulated the Bsp promoter activity mediated by Runx2 and Sox9. GATA4 knockdown by shGATA4 overexpression strongly enhanced the $B s p$ promoter activity mediated by Runx2 and Sox9 (Fig. 3C). This finding indicates that GATA4 regulates the Bsp promoter activities mediated by Runx2 and Sox9.

\section{GATA4 attenuates the binding ability of Runx2 and Sox9 to the Bsp promoter region}

To investigate the inhibitory mechanism of GATA4 on Runx2and Sox9-mediated Bsp transcriptional activity, we performed an immunoprecipitation (IP) assay to determine the interaction between GATA4 and Runx2 or Sox9. Human embryonic kidney (HEK) 293T cells were co-transfected with Flag-GATA4 and myc-Runx2 or HA-Sox9. IP assay revealed that GATA4 could directly interact with Runx2 and Sox9 (Fig. 4A, B). These findings suggest that GATA4 might inhibit the Runx2- and Sox9-mediated Bsp induction through interacting with Runx2
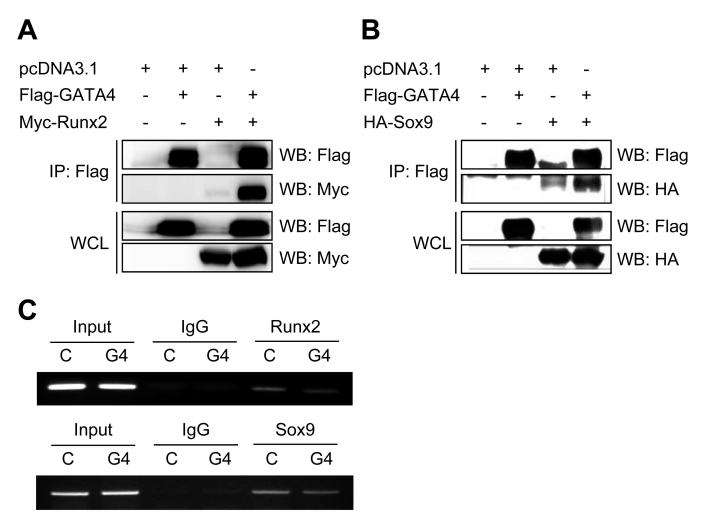

Fig. 4. GATA4 interacts with Runx2 and Sox9, and subsequently attenuates the binding ability of Runx2 and Sox9 to the Bsp promoter region. (A) HEK293T cells were transfected with FlagGATA4 and myc-Runx2. Lysates were immunoprecipitated (IP) with an anti-Flag antibody. Immunoprecipitated samples (upper panel) or whole cell lysates (WCL; lower panel) were subjected to western blotting to detect GATA4 (Flag) and Runx2 (myc). (B) HEK293T cells were transfected with Flag-GATA4 and HA-Sox9. Lysates were immunoprecipitated (IP) with an anti-Flag antibody. Immunoprecipitated samples (upper panel) or whole cell lysates (WCL; lower panel) were subjected to western blotting to detect GATA4 (Flag) and Sox9 (HA). (C) ChIP assay of GATA4 binding to the Bsp promoter region. $\mathrm{C} 2 \mathrm{C} 12$ cells were transfected with pMX-IRES-EGFP (C) or pMX-IRES-GATA4-EGFP (G4) plasmid. After 48 hours of transfection, samples were immunoprecipitated with control IgG or anti-Runx2 antibody (upper panel), and control IgG or anti-Sox9 antibody (lower panel); samples were subjected to PCR amplification with primers specific for the Runx2 binding sites (upper panel) or Sox9 binding sites (lower panel) in the Bsp promoter region. 
and Sox9.

To determine whether GATA4 could modulate the binding ability of Runx2 and Sox9 to the Bsp promoter region, we carried out a chromatin immunoprecipitation (ChIP) assay. GATA4 was transfected in $\mathrm{C} 2 \mathrm{C} 12$ cells and immunoprecipitated with Runx2 and Sox9 antibodies. ChIP assay revealed that the binding ability of Runx2 and Sox9 to the Bsp promoter region was decreased by GATA4 overexpression, than that seen in the control (Fig. 4C). Taken together, these results suggest that GATA4 can interact with Runx2 and Sox9, and attenuates the binding ability of Runx2 and Sox9 to the Bsp promoter region.

\section{DISCUSSION}

In the present study, we analyzed the regulatory mechanism of $B s p$ by various transcription factors, which are important for osteoblast differentiation. Our data showed that Bsp, one of the important genes in osteoblasts, was strongly activated by overexpression of Runx2, Dlx5, and Sox9. Bsp activation by these transcription factors was attenuated by GATA4, suggesting that GATA4 negatively regulates Bsp expression during osteoblast differentiation.

GATA4 had been studied in cardiovascular development such as maintenance of postnatal cardiac function and protection from stress-induced heart failure. GATA4 is responsible for pathological cardiac hypertrophy. We previously demonstrated that GATA4 plays a negative role in osteoblast differentiation by down-regulating the osteogenic genes such as Runx2, ALP,Bsp, and OCN. GATA4 interacts with Dlx5 and subsequently inhibits the binding ability of Dlx5 to the Runx2 promoter region (22). Taken together, our results suggest that GATA4 negatively regulates osteoblast differentiation via down-regulation of Runx2 and Bsp. Further studies are warranted to determine whether GATA4 regulates other osteoblastic genes.

Sox9 suppresses Runx2-mediated OCN expression (8). However, in this study, exogenous Sox9 increased Bsp expression in MC3T3-E1 and $\mathrm{C}_{3} \mathrm{H}_{10 \mathrm{~T}_{1 / 2}}$ cells. Furthermore, Sox9 augmented the $B s p$ promoter activity when using the $B s p$ promoter that contained the Sox9-response elements. In addition, Sox9 further enhanced the Runx2-mediated transactivation of the Bsp promoter. These data indicate that Sox9 might regulate the Bsp expression together with Runx2. Interestingly, Sox9 overexpression could induce Runx2 expression in MC3T3-E1 cells, suggesting that Sox9 might also indirectly regulate Bsp expression via Runx2 induction.

In summary, our study demonstrates that GATA4 might play a negative role in osteoblast differentiation, by regulating the Bsp expression via modulating the binding activities of Runx2 and Sox9 to the Bsp promoter region. We recently demonstrated that GATA4 down-regulates the Runx2 gene. Hence, our study reveals an additional layer of negative regulation of GATA4 in osteoblasts. Further elucidation of the regulatory mechanism of GATA4 for other osteoblastic genes might provide additional therapeutic approaches to various bone diseases.

\section{MATERIALS AND METHODS}

\section{Reagents}

Antibodies against Flag and hemagglutinin ( $\mathrm{HA})$ were obtained from Sigma-Aldrich (St Louis, MO, USA) and Roche Applied Sciences (Indianapolis, IN, USA), respectively. Anti-Runx2 and anti-Bsp were purchased from Santa Cruz Biotechnology, INC. (Dallas, TX, USA). Anti-Sox9 was obtained from Abcam (Cambridge, UK). Dynabead protein G for immunoprecipitation was obtained from Novex Life technologies (Carlsbad, CA, USA).

\section{Constructs}

GATA4 was prepared by RT-PCR using RNA from C57BL/6 mouse heart, as previously described (22). Sox9 was prepared by RT-PCR using RNA from $\mathrm{C} 3 \mathrm{H}_{10 \mathrm{~T}_{1 / 2}}$ cells. The primer sequences are as follows: Sox9 sense: 5'-CGG GAT CCA CCA TGA ATC TCC TGG ACC CCT TC-3'; Sox9 antisense: 5'-CCG CTC GAG CGC GGT CAG GGT CTG GTG AGC TG-3'. The amplified PCR fragments were cloned into the HA-pcDNA3.1 vector. Bsp-1.5 $\mathrm{Kb}$ promoter luciferase reporter was kindly provided by KY Lee (Chonnam National University, Gwangju, Korea).

\section{RT-PCR}

MC3T3-E1 cells were induced by $50 \mu \mathrm{g} / \mathrm{ml}$ ascorbic acid, 10 $\mathrm{mM} \beta$-glycerophosphate, and $100 \mathrm{ng} / \mathrm{ml}$ BMP-2, to assess the expression of osteogenic marker genes during osteoblast differentiation. RT-PCR was performed as previously described $(23,24)$. The primer sequences were as follows: Sox9 sense: 5'-TTC CTC CCT TAG CCA ACC TT-3' and Sox9 antisense: 5'-GGC GTC TGG ACT TTA GTT GC-3'; Runx2 sense: 5'-CCC AGC CAC CTT TAC CTA CA-3' and Runx2 antisense: 5'-CAG CGT CAA CAC CAT CAT TC-3'; Bsp sense: 5'-AAA GTG AAG GAA AGC GAC GA-3' and Bsp antisense: 5'-ACT CAA CGG TGC TGC TTT TT-3'; OCN sense: 5'-GCG CTC TGT CTC TCT GAC CT-3' and OCN antisense: 5'-ACC TTA TTG CCC TCC TGC TT-3'; $\beta$-actin sense: 5'-AAG AGC TAT GAG CTG CCT-3' and $\beta$-actin antisense: 5'-CAC AGG ATT CCA TAC CCA-3'; GAPDH sense: 5'-TGA CCA CAG TCC ATG CCA TCA CTG-3' and GAPDH antisense: 5'-CAG GAG ACA ACC TGG TCC TCA GTG-3'.

\section{Promoter assay}

For transfection of reporter plasmids, $\mathrm{C} 2 \mathrm{C} 12$ cells were plated on 24-well plates at a density of $2 \times 10^{4}$ cells/well, one day before transfection. Plasmid DNA was mixed with TransIT2020 (Mirus, Madison, WI, USA) and transfected into the cells, as per the manufacturer's protocol. After 48 hours of transfection, the cells were washed twice with PBS and then lysed in reporter lysis buffer (Promega, Madison, WI, USA). Luciferase activity was measured with a luciferase assay 
system (Promega) according to the manufacturer's instructions. Luciferase activity was measured in triplicate, averaged, and then normalized to $\beta$-galactosidase activity using o-nitrophenyl$\beta$-D-galactopyranoside (Sigma-Aldrich) as a substrate.

\section{Immunoprecipitation assay and western blot analysis}

The 293T cells were transfected with Flag-GATA4 and mycRunx2 or Flag-GATA4 and HA-Sox9 for 48 hours, washed with chilled PBS, and lysed in extraction buffer $(50 \mathrm{mM}$ Tris- $\mathrm{HCl}$ (pH 8.0), $150 \mathrm{mM} \mathrm{NaCl}, 1$ mM EDTA, 0.5\% Nonidet P-40, PMSF, and protease inhibitors). Cell lysates were immunoprecipitated with monoclonal anti-Flag and anti-HA antibodies. Cell lysates and immunoprecipitated samples were subsequently separated by SDS-PAGE and transferred to PVDF membrane (Millipore, Temecula, CA, USA). The membrane was blocked with TBS-T (10 mM Tri-HCl (pH 7.6), $150 \mathrm{mM} \mathrm{NaCl}, 0.1 \%$ Tween 20) containing 5\% skim milk, and was probed with horseradish peroxidase (HRP)-conjugated antibodies, including anti-Flag-HRP (Sigma) and anti-HA-HRP (Sigma). Signals were detected with chemiluminescence $(\mathrm{ECL})$ photo film.

\section{ChIP assay}

A chromatin immunoprecipitation (ChIP) assay was performed with a ChIP kit (Upstate Biotechnology, Lake Placid. NY, USA), according to the manufacturer's instructions, using antibodies against Sox9 and Runx2 with control IgG (Santa Cruz Biotechnology, Santa Cruz, CA, USA). The precipitated DNA was subjected to PCR amplification with specific primers for the Bsp $1.5 \mathrm{~kb}$ promoter region containing Sox9- and Runx2-binding sites. The following primers were used for PCR: Bsp-R3 sense, 5'-GCT TAT GGG GGT GAA TTG AA-3'; Bsp-R3 antisense, 5'-AGC TAA AGA AAA GTA TTT CAG CAT TT-3' for Runx2, and Bsp-S sense, 5'-AAA TGC TGA AAT ACT TTT CTT TAG CT-3'; Bsp-S antisense, 5'-TCC CCA TAT TCT TCT CTG TTG A-3' for Sox9.

\section{Statistical analysis}

All values are expressed as means \pm SD. Statistical analyses were performed using two-tailed Student's t-tests. $P$ values less than 0.05 were considered statistically significant.

\section{ACKNOWLEDGEMENTS}

This research was supported by a Grant (CRI13905-23) from Chonnam National University Hospital Biomedical Research Institute, and a Grant (2011-0023311) from the Basic Science Research Program through National Research Foundation of Korea (NRF) at the Ministry of Education, Science, and Technology.

\section{REFERENCES}

1. Kim JH and Kim N (2016) Signaling pathways in osteoclast differentiation. Chonnam Med J 52, 12-17
2. Walsh MC, Kim N, Kadono Y et al (2006) Osteoimmunology: interplay between the immune system and bone metabolism. Annu Rev Immunol 24, 33-63

3. Komori T, Yagi H, Nomura $\mathrm{S}$ et al (1997) Targeted disruption of Cbfa1 results in a complete lack of bone formation owing to maturational arrest of osteoblasts. Cell 89, 755-764

4. Otto F, Thornell AP, Crompton T et al (1997) Cbfa1, a candidate gene for cleidocranial dysplasia syndrome, is essential for osteoblast differentiation and bone development. Cell 89, 765-771

5. Lee B, Thirunavukkarasu K, Zhou L et al (1997) Missense mutations abolishing DNA binding of the osteoblastspecific transcription factor OSF2/CBFA1 in cleidocranial dysplasia. Nat Genet 16, 307-310

6. Hong JH, Hwang ES, McManus MT et al (2005) TAZ, a transcriptional modulator of mesenchymal stem cell differentiation. Science 309, 1074-1078

7. Lee MH, Kim YJ, Yoon WJ et al (2005) Dlx5 specifically regulates Runx2 type II expression by binding to homeodomain-response elements in the Runx2 distal promoter. J Biol Chem 280, 35579-35587

8. Zhou G, Zheng Q, Engin F et al (2006) Dominance of SOX9 function over RUNX2 during skeletogenesis. Proc Natl Acad Sci U S A 103, 19004-19009

9. Marie PJ (2008) Transcription factors controlling osteoblastogenesis. Arch Biochem Biophys 473, 98-105

10. Gubbay J, Koopman P, Collignon J, Burgoyne P and Lovell-Badge R (1990) Normal structure and expression of Zfy genes in XY female mice mutant in Tdy. Development $109,647-653$

11. Jo A, Denduluri S, Zhang B et al (2014) The versatile functions of Sox9 in development, stem cells, and human diseases. Genes Dis 1, 149-161

12. Ikeda T, Kamekura S, Mabuchi A et al (2004) The combination of SOX5, SOX6, and SOX9 (the SOX trio) provides signals sufficient for induction of permanent cartilage. Arthritis Rheum 50, 3561-3573

13. Molkentin JD (2000) The zinc finger-containing transcription factors GATA-4, -5 , and -6 . Ubiquitously expressed regulators of tissue-specific gene expression. J Biol Chem 275, 38949-38952

14. Viger RS, Guittot SM, Anttonen M, Wilson DB and Heikinheimo M (2008) Role of the GATA family of transcription factors in endocrine development, function, and disease. Mol Endocrinol 22, 781-798

15. Pikkarainen $\mathrm{S}$, Tokola $\mathrm{H}$, Kerkela $\mathrm{R}$ and Ruskoaho $\mathrm{H}$ (2004) GATA transcription factors in the developing and adult heart. Cardiovasc Res 63, 196-207

16. Foster BL, Soenjaya Y, Nociti FH Jr et al (2013) Deficiency in acellular cementum and periodontal attachment in bsp null mice. J Dent Res 92, 166-172

17. Ganss B, Kim RH and Sodek J (1999) Bone silaoprotein. Crit Rev Oral Biol Med 10, 79-98

18. Fisher LW and Fedarko NS (2003) Six genes expressed in bones and teeth encode the current members of the SIBLING family of proteins. Connect Tissue Res 44, 33-40

19. Hunter GK and Goldberg HA (1994) Modulation of crystal formation by bone phosphoproteins: role of glutamic acid-rich sequences in the nucleation of hydroxyapatite by 
bone sialoprotein. Biochem J 302, 175-179

20. Malaval L, Wade-Guéye NM and Boudiffa M et al (2008) Bone sialoprotein plays a functional role in bone formation and osteoclastogenesis. J Exp Med 205, 1145-1153

21. Barnes GL, Javed A, Waller SM et al (2003) Osteoblastrelated transcription factors Runx2 (Cbfa1/AML3) and MSX2 mediate the expression of bone sialoprotein in human metastatic breast cancer cells. Cancer Res 63, 2631-2637

22. Song I, Kim K, Kim JH et al (2014) GATA4 negatively regulates osteoblast differentiation by downregulation of Runx2. BMB Rep 47, 463-468

23. Hu L, Su P, Li R et al (2015) Knockdown of microtubule actin crosslinking factor 1 inhibits cell proliferation in MC3T3-E1 osteoblastic cells. BMB Rep 48, 583-588

24. Kim K, Kim JH, Lee J et al (2005) Nuclear factor of activated T cells $\mathrm{c} 1$ induces osteoclast-associated receptor gene expression during tumor necrosis factor-related activation-induced cytokine-mediated osteoclastogenesis. J Biol Chem 280, 35209-35216 